\title{
Mineralocorticoid receptor is essential for corticosteroid-induced up-regulation of L-type calcium currents in cultured neonatal cardiomyocytes
}

\author{
Jean-Sébastien Rougier • Olivier Muller • \\ Stefan Berger • Gabriel Centeno • Günther Schütz • \\ Dmitri Firsov • Hugues Abriel
}

Received: 13 August 2007 / Accepted: 31 October 2007 / Published online: 27 November 2007

(C) Springer-Verlag 2007

\begin{abstract}
Despite the fact that mineralocorticoid receptor (MR) antagonist drugs such as spironolactone and eplerenone reduce the mortality in heart failure patients, there is, thus far, no unambiguous demonstration of a functional role of MR in cardiac cells. The aim of this work was to investigate the activation pathway(s) mediating corticosteroid-induced upregulation of cardiac calcium current $\left(I_{\mathrm{Ca}}\right)$. In this study, using neonatal cardiomyocytes from MR or glucocorticoid receptor (GR) knockout (KO) mice, we show that MR is essential for corticosteroid-induced up-regulation of $I_{\mathrm{Ca}}$. This study provides the first direct and unequivocal evidence for MR function in the heart.
\end{abstract}

Keywords Corticosteroids · Nuclear receptors .

Aldosterone $\cdot$ Calcium current

J.-S. Rougier $\cdot$ G. Centeno $\cdot$ D. Firsov $(\bowtie) \cdot H$. Abriel

Department of Pharmacology and Toxicology,

University of Lausanne,

Bugnon, 27,

1005 Lausanne, Switzerland

e-mail: Dmitri.Firsov@unil.ch

O. Muller $\cdot$ H. Abriel

Service of Cardiology, CHUV,

Lausanne, Switzerland

S. Berger $\cdot$ G. Schütz

German Cancer Research Center,

Division Molecular Biology of the Cell I,

Im Neuenheimer Feld 280,

69120 Heidelberg, Germany

H. Abriel $(\square)$

Department of Pharmacology and Toxicology,

and Service of Cardiology, University of Lausanne,

Bugnon, 27,

1005 Lausanne, Switzerland

e-mail: Hugues.Abriel@unil.ch

\section{Introduction}

Adrenal corticosteroids [mineralocorticoids (MR) and glucocorticoids (GR)] target many effector organs, including the heart. The natural mineralocorticoid aldosterone and the glucocorticoid cortisol (corticosterone in rodents) bind to intracellular receptors, the high affinity MR, and the low affinity GR, which are both expressed in cardiomyocytes [2]. However, cardiomyocytes are defined as "nonclassical" MR-expressing cells because the ligand specificity (aldosterone vs cortisol/corticosterone) of the receptor remains unclear. While in classical MR-expressing tissues, such as the kidney and the colon, the MR is protected from occupancy by glucocorticoids by the activity of the 11- $\beta$ hydroxysteroid-dehydrogenase type- $2(11 \beta$-HSD2) that converts cortisol/corticosterone into inactive metabolites; cardiomyocytes demonstrate very low levels of this enzyme [2]. This observation suggests that there is very little, if any, "MR-protection" mechanism, which may circumvent MR occupancy by glucocorticoids circulating at concentrations $>100$-fold higher than aldosterone. Thus, in cardiomyocytes, MR is most likely occupied only by glucocorticoids.

In the past few years, the beneficial effect of MR antagonist drugs such as spironolactone and eplerenone in heart failure patients has been clearly demonstrated [8]. However, the molecular mechanisms underlying these clinical observations remain poorly understood. In particular, the role of MR activation or blockade in the heart is a matter of debate. One of the key questions that have been difficult to address is to what extent MR-mediated effects can be dissociated from GR-dependent effects.

In the present study, we addressed whether MR or GR activation is responsible for corticosteroid-induced upregulation of $I_{\mathrm{Ca}}$ [1] using MR or GR knockout mice. Our experiments unequivocally demonstrate the essential role of 
MR in mediating the corticosteroid-dependent up-regulation of $I_{\mathrm{Ca}}$, thus providing the first demonstration of a functional role of MR in cultured neonatal cardiac cells.

\section{Materials and methods}

\section{Cell culture}

Due to the early lethality of the MR and GR knockout mice, the hearts (atria and ventricles) from newborn mice or from mouse fetuses (day 18.5 of gestation, male and female) were excised under sterile conditions and cut into four equal parts. After extensive washing, these parts were transferred into an Eppendorf tube containing $1 \mathrm{ml}$ of Hanks' medium (Gibco/Invitrogen), supplemented with $0.5 \mathrm{mg} / \mathrm{ml}$ trypsin (USB). Incubation with trypsin lasted overnight with gentle agitation $(700 \mathrm{rpm}$, Eppendorf Compact Thermomixer) at $4^{\circ} \mathrm{C}$. At the end of the incubation with trypsin, tissue was allowed to settle for $1 \mathrm{~min}$, and the 500- $\mu$ l trypsinization solution was removed and replaced by $500 \mu \mathrm{l}$ of Light medium composed of Dulbecco's modified Eagle's medium (DMEM)/M-199 media $(3 / 1, v / v)$, supplemented with $1 / 100$ times diluted $(v / v)$ standard mix of penicillin/streptomycin/glutamine (Gibco/Invitrogen), and $10 \mathrm{mM}$ Hepes ( $\mathrm{pH}$ 7.3). Tubes were incubated for $2.5 \mathrm{~min}$ with gentle agitation (1,100 rpm, Eppendorf Compact Thermomixer) at $37^{\circ} \mathrm{C}$. Tissue was allowed to settle, and medium was replaced by $250 \mu \mathrm{l}$ of Hanks' medium, supplemented with $300 \mathrm{U} / \mathrm{ml}$ collagenase type 2 (Worthington Biochemical). Tubes were incubated for 1.5 min with gentle agitation $(1,100 \mathrm{rpm}$, Eppendorf Compact Thermomixer) at $37^{\circ} \mathrm{C}$. Medium was discarded and replaced by a new $250-\mu$ l aliquot of Hanks'/ collagenase solution. Tubes were incubated for $7 \mathrm{~min}$ at $37^{\circ} \mathrm{C}$ (1,100 rpm, Eppendorf Compact Thermomixer). Medium was removed and mixed with $250 \mu$ of Complete medium composed of the Light medium supplemented with $10 \%$ horse serum and 5\% fetal calf serum (Gibco/Invitrogen). The collagenase digestion step was performed two additional times. The three fractions of cardiac cells were centrifuged separately at $750 \times g$ for $5 \mathrm{~min}$ at $4{ }^{\circ} \mathrm{C}$. Medium was discarded and cell pellets were pooled in $750 \mu \mathrm{l}$ of Complete medium. Cells were plated in a single well of a 24 -well plate and incubated for $1 \mathrm{~h} 15 \mathrm{~min}$ at $37^{\circ} \mathrm{C}\left(10 \% \mathrm{CO}_{2}\right)$. This step allows attachment of fibroblasts to the well surface and their separation from other cells. This step was repeated once. The remaining cells were plated in two wells of a 24-well plate coated with gelatin and fibronectin. In the resulting culture, at least $85 \%$ of all cells were cardiomyocytes. The cardiomyocytes were actively beating throughout the experimental period (3 days). The MR(null/null) and GR(null/null) neonatal mice were obtained by breeding of corresponding heterozygous mice bearing one null and one wild-type allele [5]. All animal experiments were carried out according to the Swiss Animal Protection Law.

\section{Electrophysiological procedures}

Patch-clamp recordings in whole cell configuration were carried out using an internal solution containing ( $\mathrm{mmol} / \mathrm{l})$ : CsAsp 70, MgATP 5, CsCl 60, EGTA 11, HEPES 10, $\mathrm{MgCl}_{2}$ 1, pH 7.2 with $\mathrm{CsOH}$; external solution: TEA-Cl 130, $\mathrm{CaCl}_{2} 2, \mathrm{MgCl}_{2}$ 1.2, CsCl 5, HEPES 10, Glucose 5, $\mathrm{pH} 7.4$ with $\mathrm{CsOH}$. The resistance of the pipettes was in the range of 1.7-2.5 $\mathrm{M} \Omega$. Experiments were performed at RT $\left(22-23^{\circ} \mathrm{C}\right)$. A voltage-gated sodium channel blocker, tetrodotoxin $30 \mu \mathrm{M}$, was added in the bath before the recording of calcium current. Finally, to investigate the different biophysical properties of the calcium channel, the activation and the steady-state inactivation protocols were performed.

\section{Drugs}

Tetrodotoxin was purchased from alomone (Jerusalem, Israel). Aldosterone, corticosterone, fibronectin, and gelatine type A were from Sigma (Buchs, Switzerland). Trypsin, HBSS (ref 14170-070), DMEM, M-199, penicillin/streptomycin/glutamine and HEPES were from Gibco (Basel, Switzerland). Collagenase type II was purchased from Worthington Biochemical (Allschwil, Switzerland).

\section{Quantitative PCR analyses}

Primary cultures of neonatal mouse cardiomyocytes were either untreated (control) or stimulated for $24 \mathrm{~h}$ with aldosterone $\left(10^{-6} \mathrm{M}\right)$. Total RNA was extracted and reverse transcribed using Superscript II reverse transcriptase (Invitrogen). Real-time quantitative polymerase chain reaction (PCR) analysis was performed using the TaqMan system from Applied Biosystems. All reactions were performed in triplicate. All primer sets used in this study were from Applied Biosystems: TaqMan Gene Expression Assay: PER1 Mm00501813 m1, $\mathrm{Ca}_{\mathrm{v}} 1.2 \mathrm{Mm00437917}$, and mouse ACTB ( $\beta$-actin) endogenous control (for $\beta$-actin).

Data analysis and statistics

Measurements were made using a VE-2 (Alembic Instruments, Montreal, QC, Canada) amplifier. Data were analyzed using pClamp software, version 8 (Axon Instruments, Union City, California, USA) and KaleidaGraph software (Synergy Software, Reading, PA). Peak currents were measured using the protocol, and $\mathrm{Ca}^{2+}$ current $\left(I_{\mathrm{Ca}}\right)$ 
density (expressed as $\mathrm{pA} / \mathrm{pF}$ ) was obtained by dividing the peak current by the cell capacitance obtained using the pClamp function. After the acquisition, currents were filtered at $25 \mathrm{~Hz}$ with low pass type Gaussian filter using pClamp software, version 8 (Axon Instruments, Union City, California, USA).

Two-tailed Student $t$ test was used to compare two groups. Statistical significance is set at $P<0.05$. Values are expressed as means \pm SEM.

\section{Results}

Aldosterone-induced increase of $I_{\mathrm{Ca}}$ depends on MR, but not GR, expression

First, we determined the effect of a 24-h incubation of $1 \mu \mathrm{M}$ aldosterone on $I_{\mathrm{Ca}}$ of neonatal mouse cardiomyocytes by performing whole-cell patch-clamp experiments (Fig. 1a). Importantly, $1 \mu \mathrm{M}$ aldosterone is predicted to occupy both MR and GR, but to distinguish between MRand GR-mediated effects, myocytes prepared from either MR- or GR-KO mice were used. The current elicited at $+10 \mathrm{mV}$ is most likely due to L-channel activation [4] (Fig. 1a). After aldosterone incubation, $I_{\mathrm{Ca}}$ was increased by $86 \pm 22 \%$ (Fig. $1 \mathrm{a}, \mathrm{b}$ and Table 1 ). In contrast, no significant increase of $I_{\mathrm{Ca}}$ currents was observed in MRKO cells $(7 \pm 17 \%$, Fig. 1c,d and Table 1). Aldosteroneinduced up-regulation of $I_{\mathrm{Ca}}$ in GR-KO cardiomyocytes was very similar to that observed using WT cells $(+86 \pm$ 13\%, Fig. 1e,f and Table 1).

Biophysical properties of $I_{\mathrm{Ca}}$ are not modified by aldosterone treatment

In parallel, $I_{\mathrm{Ca}}$ was also elicited by 200 -ms step depolarizations from a holding potential of $-100 \mathrm{mV}$. Exposure of a

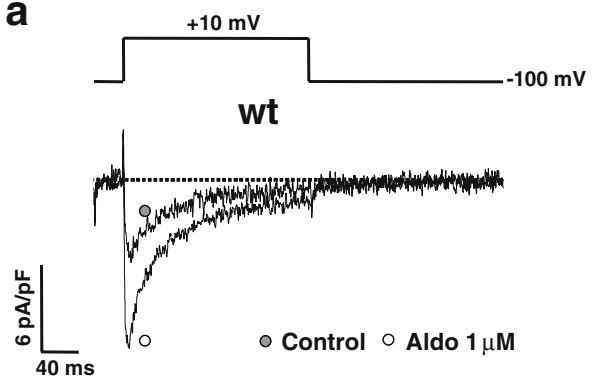

C

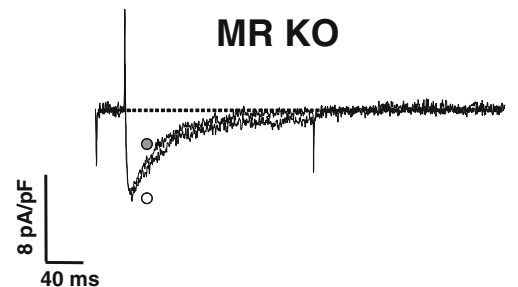

e

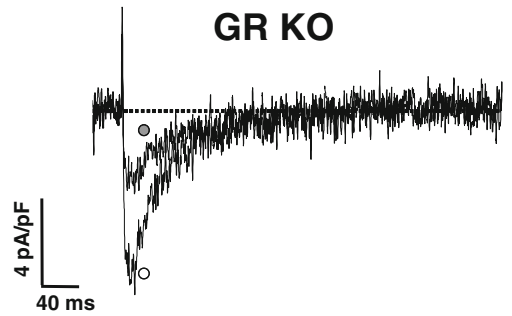

b

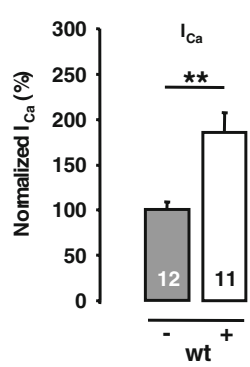

d

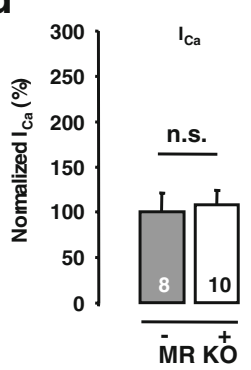

f

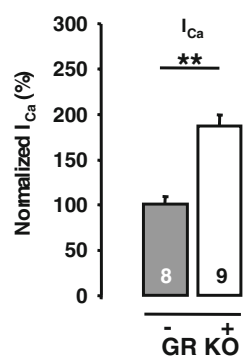

g

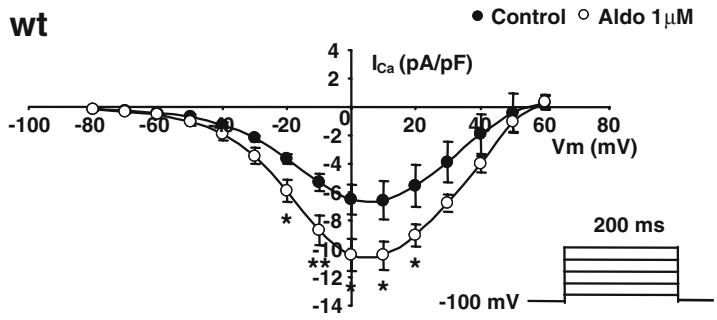

h

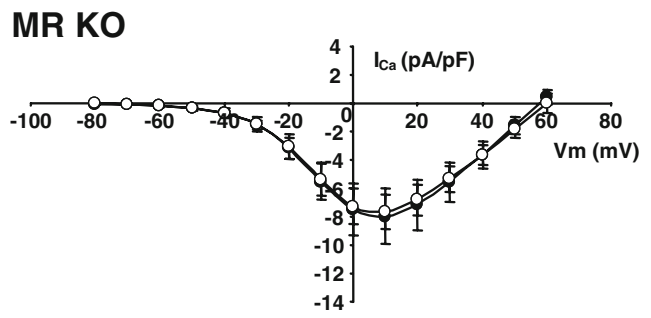

i GR KO

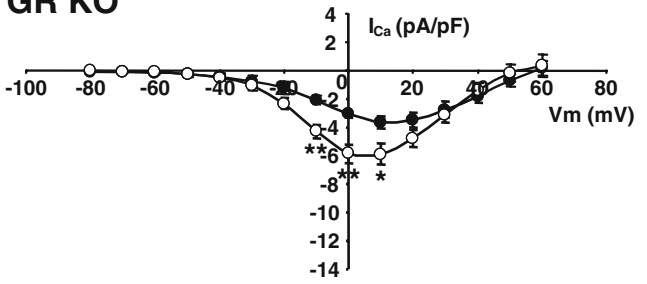

Fig. 1 Effect of aldosterone incubation on $I_{\mathrm{Ca}}$ on WT, MR, and GR$\mathrm{KO}$ myocytes. a, c, and e Holding potential was $-100 \mathrm{mV}$. Gray dots show the current from control cells, and the white dots show the current from treated cells ( $24 \mathrm{~h}, 1 \mu \mathrm{M}$ aldosterone). b, d, and f Normalized $I_{\mathrm{Ca}}$ currents determined in cells treated as indicated. The number of cells used is indicated within the bars; $* P<0.05, * * P<0.01$,

n.s. Not significant. $\mathbf{g}, \mathbf{h}, \mathbf{i}$ Modulation of $I_{\mathrm{Ca}}$ by aldosterone on wt (g) and $\mathrm{KO}$ (h and i) mouse cardiomyocytes. Current density-voltage relationships of $I_{\mathrm{Ca}}$ in control myocytes (black dots) and after aldosterone treatment $(1 \mu \mathrm{M}$ for $24 \mathrm{~h}$; white dots); $n=5-9$ cells per condition; $* P<0.05, * * P<0.01$ vs control 
Table 1 The data from individual cells were fitted with Boltzmann relationship, $y\left(V_{\mathrm{m}}\right)=1 /\left(1+\exp \left[\left(V_{\mathrm{m}}-V_{1 / 2}\right) / K\right]\right)$, in which $y$ is the normalized current or conductance, $V_{\mathrm{m}}$ is the membrane potential,
$V_{1 / 2}$ is the voltage at which half of the channels are activated or inactivated, and $K$ is the slope factor

\begin{tabular}{|c|c|c|c|c|c|c|c|}
\hline Parameter & Experiment & Wild type & & MR-KO & & GR-KO & \\
\hline \multirow[t]{2}{*}{ Cell capacitance $(\mathrm{pF})$} & Control & $11.9 \pm 1.2(n=12)$ & & $11.0 \pm 1.4(n=8)$ & & $11.1 \pm 1.4(n=8)$ & \\
\hline & Aldosterone & $11.4 \pm 0.8(n=11)$ & n.s. & $10.2 \pm 0.9(n=10)$ & n.s. & $9.9 \pm 0.7(n=9)$ & n.s. \\
\hline \multicolumn{8}{|l|}{ Activation } \\
\hline \multirow[t]{2}{*}{$K(\mathrm{mV})$} & Control & $11.8 \pm 1.1(n=8)$ & & $9.9 \pm 0.9(n=7)$ & & $10.9 \pm 2.7(n=4)$ & \\
\hline & Aldosterone & $11.3 \pm 0.9(n=9)$ & n.s. & $9.7 \pm 1.2(n=5)$ & n.s. & $8.9 \pm 0.8(n=7)$ & n.s. \\
\hline \multirow[t]{2}{*}{$V_{1 / 2}(\mathrm{mV})$} & Control & $-9.6 \pm 3.0(n=8)$ & & $-6.4 \pm 2.6(n=7)$ & & $-5.4 \pm 2.4(n=4)$ & \\
\hline & Aldosterone & $-8.2 \pm 2.2(n=9)$ & n.s. & $-7.8 \pm 1.4(n=5)$ & n.s. & $-6.9 \pm 1.1(n=7)$ & n.s. \\
\hline \multicolumn{8}{|l|}{ Inactivation } \\
\hline \multirow[t]{2}{*}{$K(\mathrm{mV})$} & Control & $9.4 \pm 0.6(n=6)$ & & $8.7 \pm 1.7(n=5)$ & & $8.2 \pm 0.7(n=6)$ & \\
\hline & Aldosterone & $7.4 \pm 1.6(n=5)$ & n.s. & $8.8 \pm 1.4(n=5)$ & n.s. & $8.8 \pm 0.8(n=8)$ & n.s. \\
\hline \multirow[t]{2}{*}{$V_{1 / 2}(\mathrm{mV})$} & Control & $-29.5 \pm 1.1 \quad(n=6)$ & & $-27.3 \pm 1.3(n=5)$ & & $-30.4 \pm 1.3(n=6)$ & \\
\hline & Aldosterone & $-28.3 \pm 1.6(n=5)$ & n.s. & $-29.2 \pm 1.3(n=5)$ & n.s. & $-31.0 \pm 0.8(n=8)$ & n.s. \\
\hline \multicolumn{8}{|l|}{$I_{\mathrm{Ca}}$ amplitude $(\mathrm{pA} / \mathrm{pF})$} \\
\hline & Control & $5.2 \pm 0.5(n=12)$ & & $7.0 \pm 1.5(n=8)$ & & $3.3 \pm 0.3(n=8)$ & \\
\hline & Aldosterone & $9.7 \pm 1.1(n=11)$ & $* *$ & $7.6 \pm 1.2(n=10)$ & n.s. & $6.1 \pm 0.4(n=9)$ & $* *$ \\
\hline
\end{tabular}

Peak $I_{\mathrm{Ca}}$ was measured at $+10 \mathrm{mV}$ during a classical protocol. Number of cells in parenthesis

n.s. Not significant vs control

$* P<0.05$

$* * P<0.01$

WT cardiomyocytes for $24 \mathrm{~h}$ to $1 \mu \mathrm{M}$ aldosterone significantly increased the inward $I_{\mathrm{Ca}}$ densities between -20 and $+20 \mathrm{mV}$ (Fig. 1g), whereas it did not modify $I_{\mathrm{Ca}}$ of MR-KO cells (Fig. 1h). In contrast, $\mathrm{I}_{\mathrm{Ca}}$ density was significantly increased between -10 and $+10 \mathrm{mV}$ in GRKO myocytes (Fig. 1i). No modification of the cell capacitance was observed in WT, MR, and GR-KO cells upon aldosterone incubation (Table 1). The voltagedependence of the steady-state inactivation and activation were similar with or without aldosterone incubation, independent of the genotype of the cardiomyocytes (Table 1).

\section{Aldosterone EC50 for $I_{\mathrm{Ca}}$ up-regulation is consistent} with MR activation

As illustrated in Fig. 2a, the effect of aldosterone on $I_{\mathrm{Ca}}$ was concentration dependent. A concentration-response analysis showed that the calculated EC50 values of the 24-h aldosterone-induced increase of $I_{\mathrm{Ca}}$ (Fig. $2 \mathrm{~b}$ ) was $2.0 \mathrm{nM}$ $\left(R^{2}=0.99\right)$. This value is close to the $\mathrm{Kd}$ values $(0.5-$ $2 \mathrm{nM}$ ) of aldosterone for the MR [2].

Effect of corticosterone and RNA expression of $\mathrm{Ca}_{\mathrm{v}} 1.2$ channel subunit

In mice, the main glucocorticoid hormone is corticosterone, whereas in humans, it is cortisol. Both hormones have a similar affinity to the MR. Therefore, it could be expected that
$I_{\mathrm{Ca}}$ is also regulated by corticosterone. Corticosterone incubation $(1 \mu \mathrm{M}, 24 \mathrm{~h})$ increased $I_{\mathrm{Ca}}(+45 \pm 9 \%)$ (Fig. $\left.2 \mathrm{c}, \mathrm{d}\right)$.

Finally, to determine if the increase of L-type $I_{\mathrm{Ca}}$ is due to an up-regulation of the mRNA encoding the $\mathrm{Ca}$ channel alpha-subunit $\mathrm{Ca}_{\mathrm{v}} 1.2-C A C N A 1 C$, qPCR analyses using specific probes have been performed. Aldosterone treatment $(1 \mu \mathrm{M}, 24 \mathrm{~h})$ did not increase the mRNA level of $\mathrm{Ca}_{\mathrm{v}} 1.2$, while the positive control gene Perl [5] was increased by greater than fivefold (Fig. 2e).

\section{Discussion}

In this study, we could unequivocally demonstrate a functional role of endogenously expressed MR in cardiac cells. This is based on two main lines of evidence. Firstly, the EC50 value for the effect aldosterone on $I_{\mathrm{Ca}}$ was $\sim 2 \mathrm{nM}$, which is within the $0.1-2 \mathrm{nM}$ range of MRdependent aldosterone effects determined in different studies [2]. Secondly, these effects were abolished in myocytes of MR-deficient mice, but conserved in GRdeficient cells. We also observed that the glucocorticoid corticosterone increased the $I_{\mathrm{Ca}}$, similarly to aldosterone. Under physiologic conditions, it is very likely that the MR is continuously occupied by the circulating glucocorticoids when one considers that (1) in cardiomyocytes, the MR is "unprotected" because of the low $11 \beta-H S D 2$ activity, (2) 
a

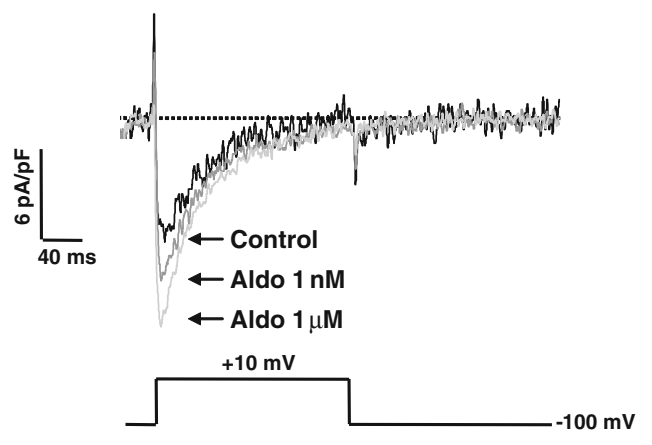

b

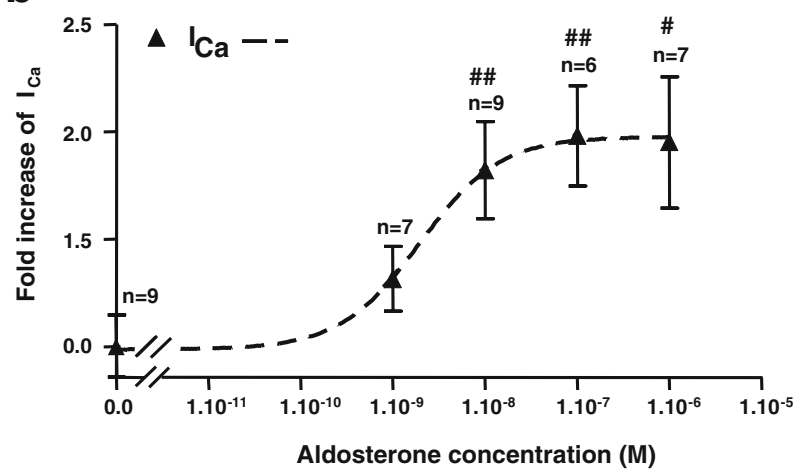

Fig. 2 Concentration-response curves of aldosterone on $I_{\mathrm{Ca}}$ of WT cardiomyocytes. a Traces of high-threshold calcium current of control and aldosterone treated $(1 \mathrm{nM}$ and $1 \mu \mathrm{M}, 24 \mathrm{~h})$ cardiomyocytes. b Concentration-response curve of aldosterone on $I_{\mathrm{Ca}}$. Data from $I_{\mathrm{Ca}}$ (dotted line) was fit according to $\%=1 / 1+((\mathrm{drug}) / \mathrm{EC} 50)) 100$ where EC50 is the drug concentration at half-maximal augmentation; $\# P<$ $0.05, \# \# P<0.01 I_{\mathrm{Ca}}$ treated vs untreated. $\mathbf{c}$ Representative traces of $I_{\mathrm{Ca}}$

$\mathrm{Kd}$ of glucocorticoids-MR interaction is within the nanomolar range, and (3) the circulating glucocorticoid concentration is $\sim 100 \mu \mathrm{M}$. However, whether the MR-glucocorticoid interaction leads to only the activation of the receptor has been recently challenged by Funder [3]. This author propose that the degree of glucocorticoid-MR complex activity may depend on the oxidation and reduction (redox) state of cells. Under metabolic stress conditions, for instance during reactive oxygen species production caused by ischemia, some yet unknown mechanisms may activate the glucocorticoidoccupied MR, which in contrast could be inactive under resting/physiologic conditions. Obviously, these putative mechanisms need to be investigated further.

There is no doubt that MR antagonist drugs are clinically effective in reducing the mortality in heart failure patients [8], by decreasing the incidence of sudden death. This observation has led to the suggestion that the electrical activity of the heart, which depends mainly on the function of ion channels, can be regulated (or dysregulated) by aldosterone. In keeping with this concept, recent studies showed that incubating isolated cardiomyocytes with high concentrations of aldosterone increased $I_{\mathrm{Ca}}[1,4]$. However,

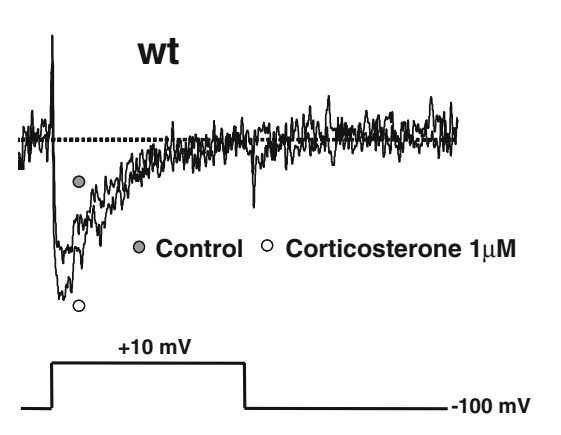

d

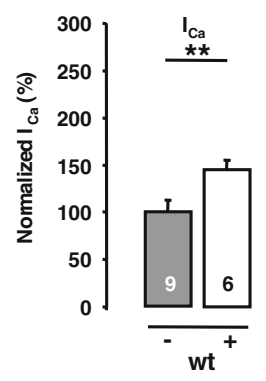

e

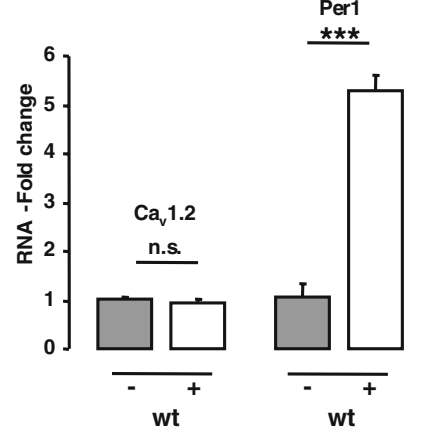

Control

$\square$ Aldo $1 \mu \mathrm{M}$

on control (gray dot) or treated with corticosterone (1 $\mu \mathrm{M}, 24 \mathrm{~h})$ (white dot). d Normalized $I_{\mathrm{Ca}}$ amplitudes determined in cells treated as indicated. The number of cells used is indicated within the bar; $* P<$ $0.05, * * P<0.01$. e RNA-fold change of $\mathrm{Ca}_{\mathrm{v}} 1.2$ and Per1 from either control (untreated) or treated (1 $\mu \mathrm{M}, 24 \mathrm{~h}$ aldosterone) cardiomyocytes. Expression levels were normalized to actin. The experiment was performed in triplicate; $* * * P<0.001$, n.s. Not significant

despite using MR and/or GR antagonists, it could never be shown convincingly that the effects of aldosterone were mediated only by the MR. Indeed in most studies, very high concentrations of aldosterone were used $(10-1,000 \mathrm{nM})$ which may activate both MR and GR. In addition, most of the drugs that were used are not completely specific for either receptor. Here, using myocytes from GR or MR-KO mice, the essential role of MR in mediating the corticosteroid-induced increase of $I_{\mathrm{Ca}}$ could be demonstrated. These findings, in combination with our recent work showing that the induction of the enzyme Art3 by corticosteroids depends exclusively on GR [5], demonstrate that the two receptor-mediated pathways may function in parallel in cardiomyocytes.

How may these results influence our understanding of the positive effect of MR antagonist drugs [8]? In mice, it has been shown that high aldosterone concentrations and cardiac-specific over-expression of MR prolong the duration of action potentials [7] and QT intervals of the ECG [6]. This phenotype is most likely the result of a MRdependent electrical remodeling process leading to an acquired form of long QT syndrome. It may be speculated that MR antagonists are beneficial and, in particular, reduce 
the rate of sudden death because they interfere with this deleterious electrical remodeling process.

In conclusion, the findings of this study demonstrate the essential role of MR in aldosterone-induced up-regulation of $I_{\mathrm{Ca}}$. However, extrapolation to the in vivo and clinical setting is limited, as we have no information about the role of MR activation by glucocorticoids. Moreover, one has to be cautious when trying to extrapolate such results, as they have been obtained from experiments performed using cultured neonatal cardiac cells that are obviously not recapitulating the in vivo or adult complexity. More experiments will be needed to understand further the multiple mechanisms underlying the beneficial effects of MR-antagonist drugs on the occurrence of sudden cardiac death.

Acknowledgments This study has been funded by the Swiss National Science Foundation PP00-110638/1 (HA) and 3100AO105592 (DF). We are grateful to Dr. Michael Harris for his helpful suggestions on this manuscript.

\section{References}

1. Benitah JP, Vassort G (1999) Aldosterone upregulates $\mathrm{Ca}(2+)$ current in adult rat cardiomyocytes. Circ Res 85:1139-1145

2. Firsov D, Muller OG (2003) Aldosterone action in the heart. Pflugers Arch 446:328-333

3. Funder JW (2005) RALES, EPHESUS and redox. J Steroid Biochem Mol Biol 93:121-125

4. Lalevee N, Rebsamen MC, Barrere-lemaire S, Perrier E, Nargeot J, Benitah JP et al (2005) Aldosterone increases T-type calcium channel expression and in vitro beating frequency in neonatal rat cardiomyocytes. Cardiovasc Res 67:216-224

5. Muller O, Pradervand S, Berger S, Centeno G, Milet A, Nicod P et al (2007) Identification of corticosteroid-regulated genes in cardiomyocytes by serial analysis of gene expression. Genomics $89: 370-377$

6. Ouvrard-Pascaud A, Sainte-Marie Y, Benitah JP, Perrier R, Soukaseum C, Nguyen Dinh Cat A et al (2005) Conditional mineralocorticoid receptor expression in the heart leads to lifethreatening arrhythmias. Circulation 111:3025-3033

7. Perrier R, Richard S, Sainte-Marie Y, Rossier BC, Jaisser F, Hummler E et al (2005) A direct relationship between plasma aldosterone and cardiac L-type $\mathrm{Ca}^{2+}$ current in mice. J Physiol (Lond) 569:153-162

8. Pitt B (2005) The role of aldosterone blockade in patients with heart failure. Heart Fail Rev 10:79-83 